Esta obra está bajo una Licencia Creative Commons Atribución-NoComercial-Compartirlgual 4.0 Internacional

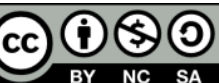

Mediación, conflictividad, negociación: Usos y apropiaciones de las TICs en la vinculación de los jóvenes con la institución escolar

Juan Matías Sterkel, Emiliano Rimoldi y Yasmín Khoury

DOI: https://doi.org/10.24215/16696581e218

\title{
Mediación, conflictividad, negociación: Usos y apropiaciones de las TICs en la vinculación de los jóvenes con la institución escolar
}

\section{Mediation, conflict ,negotiation: Uses and appropriations of ICTs in the connection of young people with the school institution}

\author{
Juan Matías Sterkel matias.sterkel@gmail.com \\ https://orcid.org/0000-0002-4516-811X
}

Facultad de Periodismo y Comunicación Social; Universidad Nacional de La Plata (Argentina)

$$
\begin{aligned}
& \text { Emiliano Rimoldi emirimoldi@gmail.com } \\
& \underline{\text { https://orcid.org/0000-0003-0858-4509 }}
\end{aligned}
$$

Facultad de Periodismo y Comunicación Social; Universidad Nacional de La Plata (Argentina)

$$
\begin{aligned}
& \text { Yazmín Khoury khoury yasmin@yahoo.com } \\
& \text { https://orcid.org/0000-0003-2454-0258 }
\end{aligned}
$$

Facultad de Periodismo y Comunicación Social; Universidad Nacional de La Plata (Argentina) 
Juan Matías Sterkel, Emiliano Rimoldi, Yasmín Khoury Mediación, conflictividad, negociacipon: Usos y apropiaciones de las TICs en la vinculación de los jóvenes con la institución escolar

\section{Resumen}

En el presente artículo se busca identificar cómo las Tecnologías de la Información y la Comunicación (TICs) reconfiguran las identidades de los sujetos, generando nuevos modos de leer y de escribir, nuevas formas de representar y reproducir/resistir la cultura; y cómo éstas se relacionan con los actores institucionales en la Escuela. En otras palabras, se analiza los diversos usos y concepciones de las TICs por parte de los sujetos y las tensiones que generan estas prácticas dentro de la institución escolar. Se diseñó una estrategia metodológica cualitativa, utilizando la modalidad de taller y herramientas como la observación participante y la entrevista en profundidad. La Unidad de Referencia Empírica (URE) estuvo constituida por estudiantes del 6to año, docentes y autoridades de la Escuela №43 de La Plata a lo largo del ciclo lectivo 2012. Se concluyó que los usos de las TICs permitidos por la institución escolar están signados por una mirada instrumental, en tanto herramientas que permiten economizar en recursos y dinamizar la labor educativa, donde el conocimiento legitimo es solo propiedad de la escuela, reforzando la relación poder-saber. De tal manera, se invisibiliza el mundo cultural de los jóvenes y las nuevas formas de socialidad, mediadas por las TICs.

Palabras clave: Tecnologías de la Información y la Comunicación (TICs); educación comunicación; poder; escolarización.

\section{Abstract}

In this article we seek to identify how ICTs reconfigure the identities of subjects, generating new ways of reading and writing, new ways of representing and reproducing / resisting culture; and how they relate to the institutional actors in the School. In other words, the diverse uses and conceptions of ICTs by the subjects and the tensions generated by these practices within the school institution are analyzed. A qualitative methodological strategy was designed, using the workshop modality and tools such as participant observation and in-depth interview. The Unit of Empirical Reference (URE) was constituted by students of the 6th year, teachers and authorities of School No. 43 of La Plata throughout the school year 2012. As a result, it was concluded that the uses of ICTs allowed by the school institution are marked by an instrumental look, as tools that allow resources to be economized and to boost educational work, where legitimate knowledge is the sole property of the school, reinforcing the power-to-know relationship. In this way, the cultural world of young people and the new forms of sociality, mediated by ICTs, become invisible.

Question, Vol. 1, N. ${ }^{\circ} 64$, octubre-diciembre 2019. ISSN 1669-6581

Instituto de Investigaciones en Comunicación | Facultad de Periodismo y Comunicación Social | Universidad Nacional de La Plata La Plata | Buenos Aires | Argentina

Página 2 de 15 
Keywords: Information and Communication Technologies (ICTs); education; communication ; power; schooling.

El programa Conectar Igualdad fue tal vez la apuesta más ambiciosa de políticas estatales en torno a las Tecnologías de la Información y la Comunicación en la Educación Secundaria. Mediante este proyecto se entregaron, entre los años 2010 y 2015, un total aproximado de cinco millones de computadoras portátiles a alumnos, docentes y directivos. El Programa se aplicó en escuelas secundarias de gestión estatal de todo el país, en todas sus modalidades; así como en las escuelas rurales, de educación especial, de educación domiciliaria y hospitalaria, y los Institutos de Formación Docente.

Producto de esa realidad, resultó la investigación desarrollada entre 2011 y 2013 realizada para la tesis de grado de la Licenciatura de Comunicación Social (Facultad de Periodismo y Comunicación Social, UNLP) "Entre lo viejo que no termina de morir y lo nuevo que no termina de nacer: TICs en tiempos de escolarización. El caso de la Escuela № 43 de La Plata".

Nuestro objetivo fue trabajar las Tecnologías de la Información y la Comunicación (TICs) desde una perspectiva de Comunicación/Educación. La intención fue aproximarnos a la temática desde un posicionamiento que evite los reduccionismos tecnócratas o tecnófobos que hegemonizaron, en ambos extremos, las investigaciones sobre las TICs en el campo de la Comunicación.

Desde la academia, las TICs, supusieron el desafío de entender tanto las formas en que se involucraban con el espacio escolar, como así también las posibilidades en torno a la educación. Superando los reduccionismos instrumentales que solo hacen foco en los aparatos tecnológicos, aparecen las perspectivas ligadas a la educomunicación. Estos estudios focalizan su atención en las prácticas que los sujetos tienen el mundo virtual y proponen darle densidad pedagógica a la vinculación entre tecnologías y educación, ya sea a través de la alfabetización multimedial (Gutiérrez Marin, 2010) o los dispositivo on-line (Quiroz Velazco, 2010).

Aun así, no profundizan en las significaciones y sentidos con la que los sujetos impregnan esas prácticas y cómo estas se sedimentan en la subjetividad y la identidad. La escuela, aun 
cuando han abrazado - no sin limitaciones - los usos de las TICs, siguen proyectando un modelo de sujeto capaz de desenvolverse en sociedad de carácter ahistórico.

Nos propusimos, entonces, abordar este tema no desde los dispositivos, sino desde los sujetos. Recuperar sus prácticas, sus concepciones, sus voces, sus experiencias; mediadas por usos, rituales y nociones en torno a las TICs. Por esta razón hablamos de procesos de mediación tecnológica, entendida como mediación cultural, que constituyen la subjetividad de los y las jóvenes y las instancias de comunicación entre ellos y los docentes y autoridades.

\section{Comunicación/Educación: Hacia una teoría transdisciplinar}

El campo de Comunicación/Educación surge desde un enfoque transdisciplinar, con el objetivo de abordar una serie de problemáticas que no podrían ser resueltas desde las dos disciplinas por separado.

Este intercambio entre disciplinas es conceptualizado como articulación, entendida como una relación en la cual ambos elementos se ven modificados sustancialmente en su identidad. Según Jorge Huergo (2001a), la propuesta de sustituir la cópula "y", de Comunicación "y" Educación, por la barra "/": Comunicación/Educación, tiene como propósito significar la recuperación de procesos, situados en contextos socioculturales y políticos, a costa de la posible ruptura con los "imperialismos" de las disciplinas.

Una cuestión clave a la hora de describir este campo consiste en dejar afuera los reduccionismos que piensan a la Comunicación ligada sólo a los medios y a la Educación vinculada unívocamente con la escuela. Al referirse a esta problemática, Eva Da Porta (2004:4) plantea que en nuestro país hay una fuerte preeminencia de la Comunicación, a la cual se remite por medio de diversos significados; quedando la

Educación relegada al ámbito escolar, puesto en crisis durante las últimas décadas.

Para entender a lo educativo retomamos a Rosa N. Buenfil Burgos (1993):

"Lo que concierne específicamente a un proceso educativo consiste en que, a partir de una práctica de interpelación, el agente se constituya como un sujeto de educación activo incorporando de dicha interpelación algún nuevo contenido valorativo, conductual, conceptual, etc., que modifique su práctica cotidiana en términos de una transformación o en términos de una reafirmación más fundamentada" (p.193). Esto implica pensar en la dimensión de lo identitario, en tanto "el discurso, en la medida en que es constitutivo de lo social, es el terreno de constitución de los sujetos, es el lugar desde el cual se proponen modelos de identificación, es la constelación de significaciones compartidas que organizan las identidades sociales."

Question, Vol. 1, N. ${ }^{\circ}$ 64, octubre-diciembre 2019. ISSN 1669-6581

Instituto de Investigaciones en Comunicación | Facultad de Periodismo y Comunicación Social | Universidad Nacional de La Plata La Plata | Buenos Aires | Argentina

Página 4 de 15 
Por otra parte, la perspectiva semiótica y de los estudios del discurso aportan a comprender la comunicación desde una perspectiva que supera el reduccionismo vinculado a lo mediático. Eliseo Verón (1987) plantea que el sentido de los discursos depende de determinadas condiciones de producción y determinadas condiciones de reconocimiento de los mismos. Es decir que el sentido no es una propiedad intrínseca de cada discurso, sino que es atribuido socialmente por quienes interpretan ese discurso.

Es necesario entender a este proceso de producción social de sentidos mediado por la cultura, entendida como una dimensión constitutiva de todas las prácticas y representaciones sociales. Jesús Martín Barbero(1987) concibe a la cultura como mediación de todos los procesos sociales, dentro de los cuales se incluye a la comunicación. De modo que puede entenderse a la mediación como la instancia cultural desde la cual los significados y sentidos son producidos y apropiados por los receptores.

Al hablar de lo cultural no nos referimos a un campo neutro, sino más bien a un terreno de disputa, donde se ponen en juego diferentes significaciones, valores, formas de ver el mundo "reconceptualizar la cultura nos enfrenta a la existencia de esa otra experiencia cultural que es la popular, en su existencia múltiple y activa y no sólo en su memoria del pasado, sino en su conflictividad y creatividad actual"(p. 227). Por lo cual es necesario retomar conceptos en torno al poder.

Para abordar el concepto de poder tomamos como base la afirmación de Michel Foucault (1998): el poder "no se da, no se intercambia ni se retoma, sino que se ejerce y sólo existe en acto"(p.23). Además, no depende de un determinismo económico, sino de relaciones de fuerza: la fuerza de la palabra, de las acciones, del discurso. Por lo tanto, el poder se construye y ejerce a través de la producción de dominios de saber y regímenes de verdad. Las nociones de derecho y verdad nos remiten a pensar en un marco de reglas que delimitan lo permitido y lo prohibido, lo que se debe hacer y lo que no se debe hacer; nos llevan a preguntarnos por los discursos y acciones postulados como legítimos en una sociedad, que se condensan en las instituciones.

La escuela es una de las múltiples instituciones que forman parte de la institución total de la sociedad. En este sentido, funciona como un espacio social que tiende a reproducir determinados discursos y regímenes de verdad. Pero además, el proyecto modernizador atribuyó a la institución escolar la función específica de imponer el saber racional sobre los saberes populares y hereditarios. Esto tiene que ver con lo que Jorge Huergo(2000) denomina el proceso de escolarización.

Este proceso está caracterizado por cuatro principios estructurales: el disciplinamiento de la vida cotidiana, el paso de la cultura oral a la lógica escritural, el desplazamiento de la cultura 
"popular" por la cultura "culta" o "letrada" y el reemplazo del "estado de naturaleza" por la vida de la sociedad. En este sentido, los objetivos de la escolarización han sido racionalizar la vida cotidiana a través del disciplinamiento; negar o minimizar los saberes calificados como "incultos", "confusos" y "desordenados" de las culturas populares; reemplazar a estos saberes por un orden reglamentado desde la palabra escrita, imponiendo regímenes de verdad y normas, en pos de la construcción de un conocimiento homogéneo y afín a las aspiraciones de la modernidad.

La progresiva racionalización de la sociedad se inicia con la institucionalización del progreso científico y técnico: "en la medida en que la ciencia y la técnica penetran en los ámbitos institucionales de la sociedad, transformando de este modo a las instituciones mismas, empiezan a desmoronarse las viejas legitimaciones" (Habermas, 1986:3). La secularización de la sociedad y el fin de las cosmovisiones hacen que los relatos de la tradición cultural pierdan eficacia en su rol de orientar la acción humana.

Desde la perspectiva habermasiana, el capitalismo tardío encuentra carencias para recurrir a las tradicionales legitimaciones, y la ideología del libre cambio es reemplazada por un programa sustitutorio centrado en una actividad estatal que compensa las disfunciones del libre intercambio. Es decir que el Estado orienta su política a la resolución de cuestiones técnicas y no a la realización de fines prácticos. Su objetivo es evitar riesgos que puedan dañar al sistema.

A partir del dominio de la lógica técnico administrativa, los criterios de justificación del sistema son despolitizados y llevados al plano de la racionalidad instrumental. La intención es que la conciencia tecnocrática se instale en los sujetos violando un interés esencial de la existencia humana: el lenguaje.

Sin embargo lo instituido, a pesar de su pretensión de presentarse como ahistórico, surge de pujas y disputas de poder - situadas histórica y socialmente - con fuerzas instituyentes que pretenden ganar terreno e instituirse ellas mismas como lo "válido": "La sociedad en tanto que siempre instituida, es auto creación y capacidad de auto alteración, obra del imaginario radical como instituyente que se auto constituye como sociedad constituida e imaginario cada vez particularizado" (Castoriadis,1998:87). Es en este interjuego entre instituido e instituyente donde se configuran las relaciones de poder entre los sujetos.

Los cambios que se han dado en los últimos años en la sociedad requieren ampliar el análisis en relación a las TICs. Para Martín Barbero (2009) ya no se puede hablar de la tecnología como una cuestión meramente instrumental, ya que hoy ésta remite "más que a unos aparatos, a nuevos modos de percepción y de lenguaje, a nuevas sensibilidades y escrituras"(p.24)

Question, Vol. 1, N. ${ }^{\circ} 64$, octubre-diciembre 2019. ISSN 1669-6581

Instituto de Investigaciones en Comunicación | Facultad de Periodismo y Comunicación Social | Universidad Nacional de La Plata La Plata | Buenos Aires | Argentina

Página 6 de 15 
El mismo autor propone el concepto de mediación tecnológica, para problematizar cómo las TICs configuran nuevas formas y ámbitos de socialidad, otros vínculos de pertenencia, instancias de aprendizaje no formales, una reconceptualización del espacio y del tiempo, etc. Además, se genera una nueva brecha, la digital, en cuanto al acceso y a los saberes que se ponen en juego en el uso de estas tecnologías.

Retomamos para nuestra investigación el concepto de mediación, atravesado por la nueva configuración tecnológica, ya que consideramos fundamental poder entender los usos que les dan los y las jóvenes a las TICs, qué sentidos y representaciones se ponen en juego, qué identidades se constituyen en este nuevo entramado.

\section{Paradigmas, métodos y herramientas}

Decidimos emprender esta investigación desde el paradigma cualitativo dado que nuestro análisis se centra fundamentalmente en un caso particular, el de una escuela, donde trabajamos con sujetos histórica, social y culturalmente situados. Según K. B. Jensen y N.W. Jankowski (1993), el análisis cualitativo se concentra en la aparición de sus objetos analíticos en un contexto específico, opuesto a la recurrencia de elementos formalmente similares en diferentes contextos. Dicho paradigma se pregunta por el significado, cuya producción se concibe como un proceso, que se contextualiza y se integra con prácticas sociales y culturales a nivel más amplio.

El paradigma cualitativo centra la investigación en el significado, el contexto, la perspectiva holística y la cultura. Es decir, el investigador se posiciona como un sujeto que comprende una realidad particular, analiza los aspectos de la vida y formas de entender el mundo del otro social, cultural, histórica, física y económicamente-, desde una visión que comprende un todo interrelacionado y amparado en qué hace el otro, qué dice que hace y cómo lo expresa.

Se utilizaron herramientas y métodos propias de la perspectiva etnográfica. Un aspecto fundamental de la etnografía tiene que ver con lo que Rosana Guber denomina interpretación o descripción densa, que implica "reconocer los marcos de interpretación dentro de los cuales los actores clasifican el comportamiento y le atribuyen sentido" (Fernandez,2013:7). Esta descripción constituye un acto interpretativo del investigador/a, quien confecciona una conclusión en base a la articulación entre la elaboración teórica y su contacto prolongado con los sujetos en el campo.

En este escenario cobra mucha importancia el lenguaje como vehículo de las situaciones de interacción. Guber plantea que el lenguaje tiene una función performativa, dado que hace la situación de interacción y define el marco que le da sentido.

Question, Vol. 1, N. ${ }^{\circ}$ 64, octubre-diciembre 2019. ISSN 1669-6581

Instituto de Investigaciones en Comunicación | Facultad de Periodismo y Comunicación Social | Universidad Nacional de La Plata La Plata | Buenos Aires | Argentina

Página 7 de 15 
El trabajo etnográfico se llevó a cabo con alumnos, docentes y autoridades de 6to año de la Escuela $\mathrm{N}^{\circ} 43$ de La Plata. Para ello fue necesario registrar etnográficamente los usos cotidianos de las TICs por parte de docentes y alumnos, cómo son esos usos y cómo son nombrados por los sujetos. Nos preguntamos fundamentalmente por el sentido que otorgan los sujetos a estos dispositivos y por sus prácticas en relación a los mismos.

\section{Métodos y herramientas}

Se recurrió a la modalidad de taller, en tanto posibilita la construcción colectiva del conocimiento, recuperando la voz de los participantes en relación con el investigador y más aún, permite ahondar en la producción de sentidos de los sujetos desde el hacer. El taller permite no solo revelar los discursos, sino también las relaciones y posiciones corporales, disposiciones prácticas, modos de proyectar la acción, etc. (Huergo, 2001b)

Se utilizó como herramienta propia de la etnografía a la observación participante, desde la cual según Rosana Guber (2001), se intenta conjugar y sintetizar dos reduccionismos que se han presentado clásicamente en la investigación y en la etnografía: la observación entendida desde el investigador como mero espectador y la participación como un intento de pertenencia e identificación con el universo de estudio llegando a desligarse del rol de investigador. Desde esta herramienta se buscó registrar en relatorías, como los estudiantes utilizan los dispositivos tecnológicos en el horario de clases.

También se realizaron entrevistas semi estructuradas con los distintos actores institucionales (Directivos, equipos de orientación, docentes), con los que se buscará obtener la mirada institucional acerca de los usos y sentidos que los jóvenes otorgan a las redes sociales en el devenir cotidiano de la escuela.

Para el análisis, proveniente de la observación participante, las entrevistas en profundidad y las producciones de los talleres, utilizamos el "método comparativo constante" (Marradi;Archenti;Piovani, 2007:294). Según su formulación original, éste consiste en cuatro etapas:

- El primer paso consiste en comparar diferentes fragmentos de datos que son agrupados, según su referencia a una misma cuestión, bajo un rótulo en común. De allí surgieron las primeras categorías conceptuales.

- Estas categorías deben ser saturadas y sometidas a las primeras interpretaciones preliminares a partir de su articulación e integración.

- Luego se seleccionan las categorías e interrelaciones centrales, lo que lleva a la transformación, integración o eliminación de varias categorías originales. 
- Por último se determina la posibilidad de generalización de la teoría y se esboza su estructura.

Sistematización y criterios para el análisis de datos

La sistematización de la información de campo estuvo plasmada en diez categorías preliminares, elaboradas en función del grado de densidad de aparición en las tres herramientas utilizadas: la entrevista en profundidad, los talleres y la observación participante.

Estas fueron:

1. Las TICs como herramienta

2. Las TICs para comunicarse

3. Las TICs como dispositivo para la disputa de poder

4. La diferenciación entre los Medios de Comunicación para informarse y las TICs para comunicarse

5. Lo generacional

6. Interpelación

7. El rol docente

8. Valor material y valor simbólico de las TICs

9. Las TICs como reemplazo de otras herramientas

10. Ritualidad

Tanto para la definición de estas diez categorías como para su priorización, utilizamos la densidad de aparición en la sistematización del trabajo de campo. Con esto nos referimos a que indefectiblemente han quedado datos por fuera de estas categorías, dado que buscamos sistematizar los emergentes que aparecían con mayor densidad en los registros, y que de alguna forma hacían más referencia a concepciones compartidas por los sujetos.

A partir de entonces, nos propusimos dar cuenta de la relación entre lo sistematizado y las nociones teóricas. Tres razones nos han llevado a delimitar las vías principales de análisis: la primera tiene que ver con haber elegido previamente algunas categorías, en particular la que se vincula a las relaciones de poder. Si bien nos propusimos no partir de premisas para realizar la investigación, entendemos que toda relación social está atravesada por el poder, y por esta razón surgió nuestra inquietud de indagar en ese sentido.

De esto se desprende que el poder - vinculado al concepto de escolarización - sea uno de los ejes centrales del análisis, desglosado en dos aparados: uno vinculado a la relación entre lo instituido y lo instituyente, otro a las negociaciones que se dan en el plano de las normas y del conocimiento. 
La segunda razón que encauzó el análisis tiene que ver con ciertos emergentes que nos llamaron la atención observando los cuadros de sistematización . ¿Por qué la categoría TICs como herramienta aparece como una de las más densas en las entrevistas y en las producciones de los talleres - con concepciones muy similares- ,pero no así en las observaciones ? ¿Por qué aparecen, tanto en los talleres como en las observaciones, nociones sistematizadas en la categoría TICs para comunicarse que nada tienen que ver con las nociones de los docentes? Esta serie de preguntas ha estructurado otro eje central del análisis, vinculado a la noción de racionalidad instrumental - también relacionada con el proceso de escolarización - y a la propuesta habermasiana de trascender a una racionalidad comunicativa.

La tercera razón determinante del análisis estuvo vinculada a la elección de determinados emergentes y a la puesta en diálogo de determinadas categorías que nos permitieran profundizar el análisis en el sentido propuesto por nuestro objetivo general de investigación. De modo que priorizamos categorías tales como Dispositivos para la disputa de poder o Rol Docente para problematizar el interjuego entre lo instituido y lo instituyente; Comunicarse o TICs como herramienta para dar cuenta de las concepciones y usos - a veces contrapuestos de docentes y alumnos; Ritualidad y Comunicarse para profundizar en el plano de la socialidad. Además de las tres razones expuestas, somos conscientes de que esta priorización de determinadas categorías y emergentes ha sido un proceso donde entraron en juego nuestras subjetividades como investigadores, nuestras inquietudes y la decisión política de profundizar en determinadas líneas de análisis.

Por lo tanto, todo el proceso expuesto nos ha llevado a la definición de tres planos principales de análisis:

-La relación entre lo instituido y lo instituyente: ¿Qué usos están instituidos en la Escuela № 43 ? ¿Cuáles son los usos instituyentes? ¿Cómo se manifiesta, en ese sentido, la relación entre lo permitido y lo prohibido?

-Las negociaciones: ¿Qué negociaciones se dan en el plano de las normas institucionales en torno al uso de las TICs? ¿Y en la relación docente-alumno? Teniendo en cuenta la relación entre poder y saber, ¿qué ocurre con el conocimiento? ¿Es un plano más de negociación?

-La socialidad: ¿Cómo se reconfigura la experiencia social de los jóvenes a partir del uso de las TICs? ¿La institución escolar reconoce estas nuevas formas de socialidad? ¿Qué retos impone esta nueva configuración social y cultural a la escuela?

Creemos que la tercera dimensión de análisis es la que quedará más abierta a nuevas discusiones. A lo largo de este recorrido pudimos sistematizar algunos emergentes y aventurar algunas conclusiones, pero entendemos que esta temática requiere de un trabajo más 
complejo para su profundización. Nos referimos fundamentalmente a futuras investigaciones que hagan foco en la vida social de los sujetos y el rol que cumplen las TICs como instancias de mediación, tanto en las relaciones sociales entre los sujetos y de los sujetos con los espacios y grupos sociales; como en los procesos de formación fuera del ámbito escolar.

\section{Conclusiones}

Al comienzo de este viaje nos propusimos indagar sobre los usos y concepciones de los docentes y alumnos de la Escuela $N^{\circ} 43$ en torno a las TICs. Llegados al final de este recorrido - lo que no es equivalente a pensar que el proceso esté agotado - quisiéramos dar cuenta de tres hallazgos centrales que nos ha dejado esta investigación. (1)

El primer hallazgo es que el uso de las TICs en la Escuela Secundaria № 43 parece estar sesgado por una mirada instrumental - al menos, en lo que refiere a usos permitidos en la institución - . La racionalidad instrumental genera que las TICs, lejos de ser consideradas como instancias de mediación cultural a partir de las cuales se constituyen nuevas subjetividades, contribuyan a reforzar la identidad de la escolarización. De modo que las TICs son consideradas como herramientas que "reemplazan el libro, la enciclopedia, el diccionario, la calculadora" (E-Di) pero cuyos usos hay que evitar o prohibir, sobre todo cuando "no tienen nada que ver con la función de la Escuela, que es educar" (E-Do)

El segundo hallazgo tiene que ver con la relación entre poder y saber: si bien hay negociaciones en el plano de las normas institucionales con respecto a los usos prohibidos de las TICs, la institución escolar no admite ninguna negociación en cuanto al conocimiento. Éste es propiedad del docente, quien debería de alguna manera "transmitir" ese conocimiento a los chicos y "guiar [pues] los alumnos tienen un bache en cuanto al contenido" dado que "conocen el circuito técnicamente [...] pero son analfabetos". (E-Do)

De este modo, la escolarización se perpetúa, en la medida en que sigue imperando en la Escuela una noción de conocimiento asociada a un modelo transmisionista y lineal de la comunicación. Un modelo que no reconoce las nuevas subjetividades y nuevas formas de socialidad, las nuevas formas de leer; que no admite como conocimiento válido a los saberes de los jóvenes.

De esto se desprende el tercer hallazgo que nos ha dejado esta investigación: la institución escolar, en su intento por imponer determinadas normas escolarizantes en torno al conocimiento y a la socialización, no efectúa un reconocimiento del mundo cultural de los jóvenes y de sus nuevas formas de socialidad, mediadas por las TICs. 
Encontramos que los y las jóvenes, en las producciones de los talleres, comparten las concepciones y usos instituidos en torno a las TICs, aquellos que nosotros identificamos atravesados por una racionalidad instrumental, por ejemplo, como herramienta "para la búsqueda de información" (T- Es) donde se evidencia cómo la institución escolar habla a través de los sujetos.

En cambio cuando preguntamos durante los talleres sobre su vida cotidiana, hallamos que los usos primordiales son otros. Notamos que los jóvenes fundamentalmente hablan de "comunicarse" con "la gente que te rodea" (T-Es) por medio del celular o del chat en las redes sociales. Notamos, también, que esos usos atraviesan la cotidianeidad de los chicxs, sus hábitos, su socialidad, sus marcos de interpretación. Es decir, son usos que atraviesan la experiencia de la vida social de los jóvenes y que se expresan en frases como: "Hay gente que no puede estar un día sin internet" (O-Es), "Si te olvidás el celu volvés a buscarlo" (T-Es), "Todos los días me conecto un 'ratito' al facebook" (T-Es) o "Una persona que no tiene facebook es extraña" (O-Es)

Esos usos, prohibidos dentro de la institución escolar, son constitutivos de la vida de los jóvenes. Creemos que este desfasaje es, en definitiva, una negación de los jóvenes como sujetos cultural e históricamente constituidos. Un no-reconocimiento de su mundo cultural, atravesado por nuevas formas de socialidad vinculadas a las TICs.

Creemos que desde el campo de la comunicación pueden hacerse aportes sumamente válidos a este debate: por un lado, concebir a la comunicación como producción social de sentidos más que como transmisión de conocimientos; por otro lado, entender que las TICs generan procesos de mediación tecnológica que constituyen tanto a los sujetos como a las instancias de comunicación.

Entender a la comunicación como producción social de sentidos alberga también la posibilidad de desjerarquizar determinados roles asumidos por docentes y directivos, en la medida en que se logre superar el modelo de comunicación escolar antes mencionado.

Pasar de una racionalidad instrumental a una racionalidad comunicativa (Habermas, 1986) implica reconfigurar a la institución escolar en su totalidad. Esta reconfiguración debe darse en dos planos. Por un lado, en el ámbito de las normas, donde las prácticas instituyentes han logrado ganar cierto terreno; por otro lado, desde el reconocimiento y la incorporación de los saberes de los jóvenes en los procesos educativos.

En lo que refiere a las normas, Habermas plantea que un proceso de racionalización en términos comunicativos estaría caracterizado por tres dimensiones de transformación: una disminución del grado de represividad, un decreciente grado de rigidez y la aproximación a un tipo de control basado en la aplicación flexible de normas bien internalizadas, pero accesibles a 
la reflexión. A este proceso se llegaría con una democratización de los ámbitos institucionales de decisión. Podemos notar que algunas de estas dimensiones se manifiestan paulatinamente en la Escuela $N^{\circ} 43$ a fuerza de prácticas instituyentes, dando lugar a negociaciones "prohibir, pero no prohibir tanto" (E - Do) - . El desafío está en abrir el juego e incorporar más actores institucionales al debate.

Por otro lado, en el plano del reconocimiento, creemos que es necesario incorporar no sólo los dispositivos tecnológicos, sino la cultura de Ixs jóvenes atravesada por las TICs. Recuperar los procesos de mediación tecnológica que van configurando nuevas formas de socialidad, nuevos gustos, nuevas costumbres, nuevos modelos de identificación. Nuevos sujetos.

La incorporación de las TICs en la Escuela debería tener por objetivo transformarla más que reformarla - que es, en definitiva, reproducirla - . El desfasaje entre la cultura escolar y la cultura de los jóvenes alberga la potencialidad de ser resuelto por medio de un uso transformador de las TICs, ligado al reconocimiento de la cultura y de los nuevos modos de leer de los y las jóvenes.

De la misma manera, si bien no hemos abordado con tanta dedicación la problemática en torno a la criticidad en el uso de las TICs, creemos que es necesario repensar esta transformación de la institución escolar vinculada a políticas educativas que promuevan el uso crítico de estas tecnologías. Las TICs por sí mismas no son transformadoras ni críticas, sino que es necesario hacer mediar la decisión política de utilizarlas en ese sentido.

Aquí se ponen en juego roles sumamente necesarios: de los directivos, de los docentes que, entendiendo a las TICs inmersas en un contexto social determinado, puedan favorecer la generación de procesos de uso y apropiación crítica y emancipadora de las tecnologías.

Por lo tanto, nos enfrentamos a dos desafíos en el plano del saber: por un lado, reconocer a los sujetos. En esto radica la posibilidad de descentralizar las relaciones de poder en lo que refiere al saber, dado que este reconocimiento trae aparejada la posibilidad de incorporar nuevos saberes - los de los jóvenes - a la Escuela.

Pero no basta con reconocer a los sujetos. No basta con incorporar sus saberes. Es también necesaria la construcción de nuevos saberes, con una perspectiva política y crítica en torno a las TICs. Y aquí surge el segundo desafío, dado que para la construcción de estos nuevos saberes es necesaria la transformación integral de la institución escolar, enmarcada en una propuesta más amplia, integral, de transformación social.

Notas

Question, Vol. 1, N. ${ }^{\circ}$ 64, octubre-diciembre 2019. ISSN 1669-6581

Instituto de Investigaciones en Comunicación | Facultad de Periodismo y Comunicación Social | Universidad Nacional de La Plata La Plata | Buenos Aires | Argentina

Página 13 de 15 
(1) Para no entorpecer la lectura se simplifican las referencias de las citas: Para los testimonios obtenidos en los talleres con estudiantes se utilizará "T-Es" y para lo registrado en fichas de observación "O-Es", cuando sea producto de entrevistas con docentes "E-Do" y si son directivos "E-Di"

\section{Bibliografía}

Buenfil Burgos, R. N.(1993) Análisis de discurso y educación. México: DIE.

Castoriadis, C.(1998) Un mundo fragmentado .La Plata: Terramar.

Da Porta, E. (2004). Senderos y recorridos. Apuntes para un mapa de investigación. La Plata.

Revista Tram[p]as (29).

Fernández, M. S. (2016). La Construcción del conocimiento etnográfico y la educación escolar.

(Trabajo final integrador). Bernal, Argentina: Universidad Nacional de Quilmes.

Recuperado de http://ridaa.unq.edu.ar/handle/20.500.11807/23

Foucault, M.(1998). Genealogía del racismo. Madrid. Caronte.

Guber, R. (2011). La Etnografía: método, campo y reflexividad. Buenos Aires: Editorial Siglo $\mathrm{XXI}$.

Gutiérrez Marin, A. (2010). Cap 8.Creación multimedia y alfabetización en la era digital. En

Roberto Aparici (comp.): Educomunicación: Mas allá del 2.0 (pp. 171-187).Barcelona: Gedisa.

Habermas, J. (1986). Ciencia y técnica como ideología. Madrid: Tecnos.

Huergo, J.(2000). "De la escolarización a la comunicación en la educación". En Huergo, Jorge; Fernández, María Belén: Cultura escolar, cultura mediática. La Plata, Universidad Pedagógica Nacional

Huergo, J. (2001a):“Comunicación y Educación: aproximaciones”, en: Huergo, Jorge (editor): Comunicación/Educación. Ámbitos, prácticas y perspectivas (pp 7-21). La Plata. Ed. de Periodismo y Comunicación.

Huergo, J. (2001b). Métodos de investigación cualitativa en comunicación. Buenos Aires. Mimeo.

Jensen, K. B.; Jankowski, N.W (1993). Metodologías cualitativas de investigación en comunicación de masas. Barcelona.Bosch

Khoury Y.;Rimoldi E.;Sterkel J.M. (2013): Entre lo viejo que no termina de morir y lo nuevo que no termina de nacer: TICs en tiempos de escolarización. El caso de la Escuela $N^{\circ} 43$ de La Plata. (Tesis de grado). La Plata, Argentina: Universidad Nacional de La Plata. Recuperado de http://sedici.unlp.edu.ar/handle/10915/72622 
Juan Matías Sterkel, Emiliano Rimoldi, Yasmín Khoury Mediación, conflictividad, negociacipon: Usos y apropiaciones

Marradi, A; Archenti, N.; Piovani, J. I. (2007). Metodología de las ciencias sociales. Buenos Aires. Emecé.

Martín Barbero, J. (1987). De los medios a las mediaciones. Comunicación, cultura y hegemonía. Barcelona. Gilli.

Martín Barbero, J. (2009). Cuando la tecnología deja de ser una ayuda didáctica para convertirse en mediación cultural. Bogotá. Revista Electrónica Teoría de la Educación(1).

Quiroz Velazco, M. T. (2010). Cap 9.Educar en otros tiempos. El valor de la comunicación. En Roberto Aparici (comp.): Educomunicación: Mas allá del 2.0 (pp. 187-205).Barcelona: Gedisa.

Verón, E.(1987): La Semiosis Social. Buenos Aires. Gedisa. 